Subscriber access provided by - Access paid by the | UCSB Libraries

\title{
Article
}

\section{Optically Trapped Surface-Enhanced Raman Probes Prepared by Silver Photo-Reduction to 3D Microstructures}

Gaszton Vizsnyiczai, Tamás Lestyán, Jaroslava Joniova, Badri Aekbote, Alena Strej\#ková, Pal Ormos, Pavol Miskovsky, Lóránd Kelemen, and Gregor Bano

Langmuir, Just Accepted Manuscript • DOI: 10.1021/acs.langmuir.5b01210 • Publication Date (Web): 20 Aug 2015

Downloaded from http://pubs.acs.org on August 24, 2015

\section{Just Accepted}

"Just Accepted" manuscripts have been peer-reviewed and accepted for publication. They are posted online prior to technical editing, formatting for publication and author proofing. The American Chemical Society provides "Just Accepted" as a free service to the research community to expedite the dissemination of scientific material as soon as possible after acceptance. "Just Accepted" manuscripts appear in full in PDF format accompanied by an HTML abstract. "Just Accepted" manuscripts have been fully peer reviewed, but should not be considered the official version of record. They are accessible to all readers and citable by the Digital Object Identifier (DOI®). "Just Accepted" is an optional service offered to authors. Therefore, the "Just Accepted" Web site may not include all articles that will be published in the journal. After a manuscript is technically edited and formatted, it will be removed from the "Just Accepted" Web site and published as an ASAP article. Note that technical editing may introduce minor changes to the manuscript text and/or graphics which could affect content, and all legal disclaimers and ethical guidelines that apply to the journal pertain. ACS cannot be held responsible for errors or consequences arising from the use of information contained in these "Just Accepted" manuscripts. 


\title{
Optically Trapped Surface-Enhanced Raman Probes
}

\section{Prepared by Silver Photo-Reduction to 3D}

\section{Microstructures}

\author{
Gaszton Vizsnyiczai, ${ }^{\dagger}$ Tamás Lestyán, ${ }^{\dagger}$ Jaroslava Joniova ${ }^{\ddagger}$ Badri L. Aekbote, ${ }^{\dagger}$ A. Strejčková, ${ }^{\S}$ \\ Pál Ormos, ${ }^{\dagger}$ Pavol Miskovsky, ${ }^{\ddagger}$ Lóránd Kelemen,$^{\dagger}$ Gregor Bánó ${ }^{*,+,}$ \\ ${ }^{\dagger}$ Biological Research Centre, Hungarian Academy of Sciences, Institute of Biophysics, \\ Temesvári krt. 62, Szeged, Hungary \\ $\$$ Department of Biophysics, Institute of Physics, Faculty of Science, P.J. Šafárik University, \\ Jesenná 5, 04101 Košice, Slovakia \\ $\S$ Department of Chemistry, Biochemistry and Biophysics, Institute of Biophysics, University of \\ Veterinary Medicine and Pharmacy, Komenského 73, 04181 Košice, Slovakia \\ " Center for Interdisciplinary Biosciences, Faculty of Science, P.J. Šafárik University, Jesenná 5, \\ 04101 Košice, Slovakia
}




\begin{abstract}
3D microstructures partially covered by silver nano-particles have been developed and tested for surface-enhanced Raman spectroscopy (SERS) in combination with optical tweezers. The microstructures made by two-photon polymerization of SU-8 photoresist were manipulated in a dual beam optical trap. The active area of the structures was covered by a SERS-active silver layer using chemically assisted photo-reduction from silver nitrate solutions. Silver layers of different grain size distributions were created by changing the photo-reduction parameters and characterized by scanning electron microscopy. The structures were tested by measuring the SERS spectra of emodin and hypericin.
\end{abstract}

\title{
INTRODUCTION
}

Optical tweezers are a robust tool for positioning and manipulating micron and sub-micron sized particles with emerging applications in the field of colloid sciences, biophysics and cell biology. During the last decade, optical tweezers were successfully combined with surfaceenhanced Raman spectroscopy (SERS), a highly sensitive technique for chemical analysis. ${ }^{1-8}$ SERS relies on local field enhancement due to plasmon resonances at nano-sized metal structures (present in colloidal suspensions ${ }^{9,10}$ or on solid SERS substrates ${ }^{11-13}$ ), which leads to manifold Raman signal enhancement from molecules located near the metal surface. ${ }^{14-17}$

There are two approaches reported in the literature for combining optical tweezers with SERS detection. First, SERS-active metal particles can be directly trapped by laser tweezers. In the work by Svedberg et al. ${ }^{1}$ a dual optical trap was used to create Ag particle dimers in a controlled way, which increased the SERS intensity by a factor of $\sim 20$. A SERS signal was also obtained from gold and silver nano-particle aggregates trapped in laser tweezers. ${ }^{2,3}$ In the second 
approach, a SERS-active metal coating is created on micron-sized carriers that are manipulated by optical forces. Silver coated microspheres were tested as SERS substrates in optical traps ${ }^{4,5}$ and were used to detect SERS spectra from yeast cells. ${ }^{6}$ Trappable SERS probes were also reported by Petrov and co-workers. Silica spheres modified with amino groups were partially covered with metal colloid particles and were used for localized drug detection in the outer cell membrane. ${ }^{7}$ Using the same probes, the recoil effect (momentum transfer) of surface-enhanced Raman photons was observed. ${ }^{8}$ Finally, these probes (even if not trapped) were applied for SERS studies of single DNA molecules, ${ }^{18}$ diffusion measurements of drugs inside of cells, ${ }^{19}$ and local $\mathrm{pH}$ detection inside of cells. ${ }^{20}$

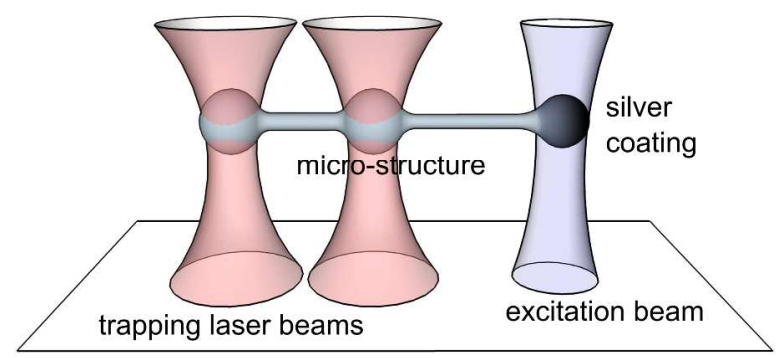

Figure 1. A microstructure composed of three connected spheres in a dual-beam optical trap. The spherical tip on the right is used for SERS detection.

The spherical SERS probes mentioned above can be prepared in a simple way and were proven to be useful in a series of experiments. However, there are some limitations connected to their applications. First, working with spherical shapes necessitates spatial overlap of the trapping and Raman excitation laser beams, which can cause unwanted heating of metal nano-particles. ${ }^{21}$ This effect can in turn lead to degradation of the studied molecules. Second, to assure transparency of the spheres (needed for successful trapping), the admissible extent of metal coverage is 
limited. ${ }^{4,7}$ The mentioned problems are eliminated using the microstructures proposed in this paper.

The novelty of the present work lies in development of optically trappable SERS sensors, where the trapping light is spatially separated from the Raman excitation. The basic idea is depicted in Figure 1 showing a microstructure composed of three connected spheres. The two spheres on the left side are used to trap the microstructure in a dual-beam optical trap, and the third one (on the right) is coated with a SERS-active metal layer. An additional laser beam is used to excite the SERS signal. The more complicated arrangement of these structures (as compared to spherical probes) puts greater demands both on the fabrication process and on the optical setup used for trapping. On the other hand, these drawbacks are compensated by having independent control of the trapping and SERS excitation laser beams. In many cases the laser power for Raman excitation can be orders of magnitude lower than the power needed for trapping. As a consequence, when working with the proposed arrangement the metal heating can be significantly reduced and the metal coverage of the SERS-active area can be increased (up to $100 \%)$

3D microstructures of arbitrary complexity designed specifically for optical tweezer applications can be fabricated by laser-assisted two-photon polymerization (TPP). ${ }^{22,23}$ We reported on 3D microstructures covered with metal nano-particles recently. ${ }^{24}$ Gold nano-particles were bound to 3D micro-tools following chemical functionalization of the used polymer, which resulted in uniform coverage of the whole microstructure surface. These systems were used for enhanced fluorescence observation but were not tested for Raman measurements. By contrast, silver photo-reduction is used in the present work to specifically cover a selected area of microstructures with a rough silver layer. The idea of making SERS-active silver nano-structures 
by photo-reduction is not new. Photo-reduced silver nano-particles were deposited by other authors onto glass substrates ${ }^{25-27}$ or silica nano-particles ${ }^{28}$ and were used for SERS detection on cellulose $^{25}$ or textile surfaces. ${ }^{29,30}$ Positively charged silver nano-particles prepared by photoreduction were also reported. ${ }^{31}$ To the best of our knowledge, this is the first time that this technique has been used for local silver deposition onto 3D microstructures.

\section{EXPERIMENTAL}

\section{Materials}

Silver nitrate $(>99 \%)$, sodium citrate tribasic dihydrate $(>99 \%)$, Triton $X-100$, emodin from frangula bark, hypericin, $\mathrm{KNO}_{3}$ and dimethyl sulfoxide (DMSO, $>99.9 \%$ ) were purchased from Sigma Aldrich. Stock solutions of emodin $\left(1.10^{-2} \mathrm{M}\right)$ and hypericin $\left(4.10^{-3} \mathrm{M}\right)$ in DMSO were prepared. The photopolymer SU-8 2007 and its developer (mrDev 600) were purchased from Microresist GmbH, Germany.

\section{Fabrication of microstructures}

The microstructures were prepared by TPP on pre-cleaned glass cover slips of $170 \mu \mathrm{m}$ thickness. $15 \mu \mathrm{m}$ thick layers of SU-8 2007 were made on the cover slips by spin-coating (Specialty Coating Systems, P-6708). The polymerization was carried out with the system described elsewhere. ${ }^{24}$ Shortly, the beam of a Ti:sapphire laser (pulse length: $100 \mathrm{fs}$, wavelength: $785 \mathrm{~nm}$, Menlo Systems, C-Fiber A) was focused into the SU-8 layer by a $100 \times$ oil immersion objective (Zeiss Achroplan, NA = 1.25). The sample was translated in 3D with piezo translators 
(Physik Instrumente, P-731.8L in X-Y and P-721.10 in Z). The applied laser power was $3 \mathrm{~mW}$ at the objective back aperture and the scan speed varied between $3 \mu \mathrm{m} / \mathrm{s}$ and $12 \mu \mathrm{m} / \mathrm{s}$.
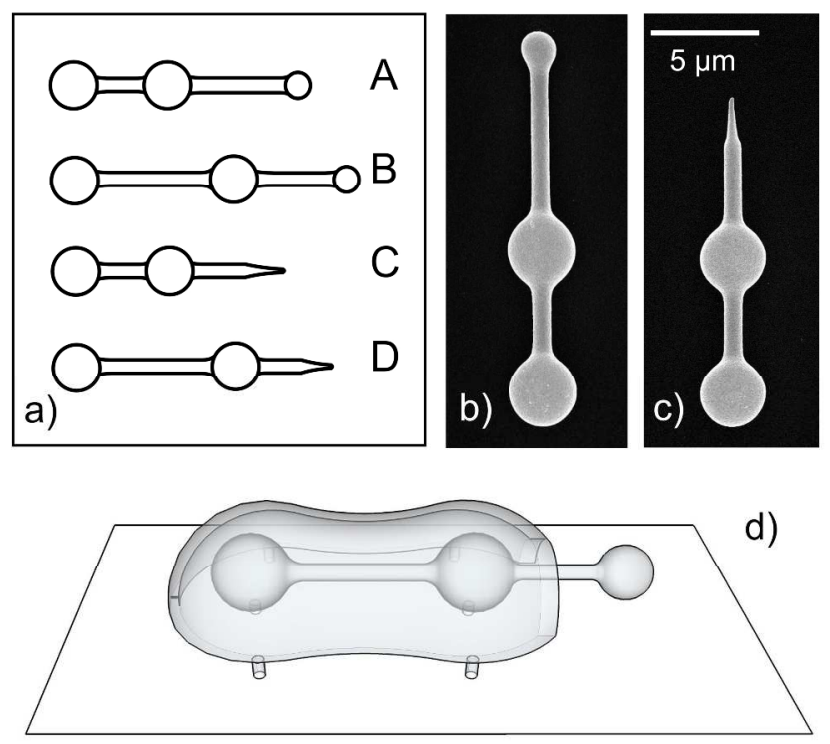

Figure 2. Microstructures fabricated by laser-assisted two-photon polymerization of SU-8 photoresist. a) Four different microstructure types with spherical $(A, B)$ and pointed $(C, D)$ tips on the right side. b) and c) SEM pictures of type-A and type-C microstructures. d) Drawing of a Type-B microstructure on glass substrate with a protective shield covering the two trapping spheres.

Four different microstructure types (assigned as A, B, C and D in Figure 2a) were tested in this work. The microstructures were composed of two connected (approx. $3 \mu \mathrm{m}$ diameter) trapping spheres and a protruding tip used for SERS measurements. The different microstructure types were prepared either with spherical $(A, B)$ or pointed $(C, D)$ tips. The distance between the two trapping spheres and the length of the tip varied. Scanning electron microscope (SEM) pictures of type-A and type-C microstructures are shown in Figure $2 \mathrm{~b}$ and $2 \mathrm{c}$. The structures were attached to the glass substrate through a pair of tiny posts located below the trapping spheres (not shown). Some of the microstructures were fabricated with a protective shield, as shown in Figure 
$2 \mathrm{~d}$. The role of the shield was to avoid metal deposition onto the trapping spheres during the photo-reduction process (see below). Again, the shield was connected to the glass slide through a set of little posts (see Figure 2d) and did not touch the microstructure itself.

Before the trapping experiments, the microstructures were submerged into water (containing $0.01 \% \mathrm{v} / \mathrm{v}$ of Triton X-100 detergent) and were examined under an inverted microscope. Individual structures were broken off the glass surface by a tipped glass needle moved with a micromanipulator. In the next step the free structures were loaded into a micropipette and were transferred to the sample cell used in the optical tweezers setup. The detergent prevented the structures from sticking to glass surfaces.

\section{The laser tweezers setup}

The microstructures were trapped and manipulated in a dual-beam optical tweezers equipped with a $785 \mathrm{~nm}$ trapping laser (New Focus, SWL-7513-H). The laser beam (shaped by spatial filtering and beam expansion) was focused into the sample by a high numerical aperture objective (Nikon Plan Fluor $100 \times, \mathrm{NA}=1.3$, oil immersion). The two traps were operated in timesharing mode. The laser beam was periodically switched (at $500 \mathrm{~Hz}$ ) between two positions using an acousto-optic deflector (AOD, Isomet LS55) placed in a plane conjugate to the back focal plane of the objective. The laser power was approx. $10 \mathrm{~mW}$ at the sample. The sample was moved by a manual XY translator stage. Fine positioning was achieved by an XY piezo stage (Physik Instrumente, P-527.2CL). The sample was illuminated by a white-light LED source from above using a long working distance $50 \times$ objective as a condenser. Trapped objects were viewed by two CCD cameras in transmitted and reflected light at higher and lower magnification, respectively. The setup was equipped with two visible lasers of $488 \mathrm{~nm}$ (Spectra Physics, Cyan) and $532 \mathrm{~nm}$ (LCS-DTL-316, Laser Compact Ltd.) wavelength coupled to the optical system 
through dichroic mirrors. These lasers were used to excite the SERS signal. The backscattered Raman signal was detected by a spectrograph (JobinYvon, iHR 550) equipped with a TE cooled CCD camera. Excitation and trapping wavelengths were blocked in front of the spectrograph by a set of notch and edge filters.
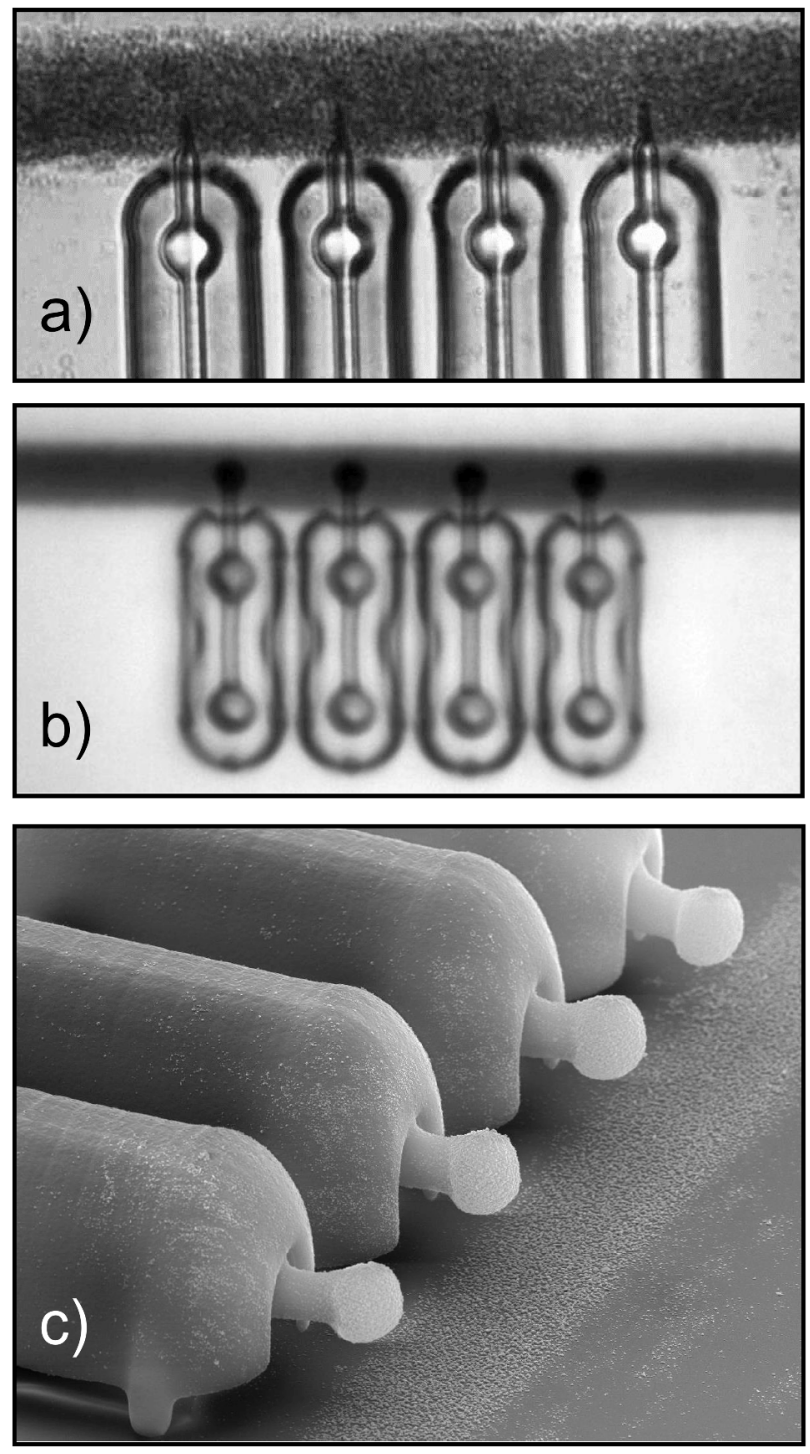

Figure 3. a) and b) Bright-field microscope pictures of Type-D and Type-B microstructures (with protective shields) on glass substrate after the photo-reduction process. The silver layer transmittance is $40 \%$. c) SEM picture of Type-B microstructures with $20 \%$ transmittance silver coating. 


\section{Silver photo-reduction}

The microstructure tips were covered by nano-structured silver layers using photo-reduction of silver nitrate solutions. The process was promoted by adding sodium citrate (a chemical reducing agent) to the solution, which increased the deposition rate. ${ }^{32}$ Prior to the photo-reduction process the solution of silver nitrate $\left(1.10^{-3} \mathrm{M}\right)$ was heated in a water bath while continuously stirring, and the sodium citrate was added (final concentration: $7.10^{-4} \mathrm{M}$ ) when its temperature reached $70^{\circ} \mathrm{C}$. Then the stirring continued for another $2 \mathrm{~min}$ and finally the mixture was cooled down to room temperature.

The coating was accomplished on-the-substrate, with microstructures still attached to the glass cover slip. A special optical setup was used for photo-reduction. The cover slip containing an array of fabricated microstructures was oriented upside-down and covered a container filled with silver nitrate solution. The microstructures immersed in the solution were irradiated from above by a $532 \mathrm{~nm}$ continuous laser $(8 \mathrm{~mW}$ at the back aperture of the objective) (Roithner Lasertechnik, CW532-50) using a 20× objective (Zeiss A-Plan, NA=0.45). A cylindrical lens $(f=200 \mathrm{~mm})$ was inserted into the optical path focusing the laser beam at the back focal plane of the objective. As a result, silver deposition took place on a thin line in the sample plane. This way, three to five neighboring microstructures could be coated in parallel (see Figure 3). During the photo-reduction process, a metal layer also built up on the glass surface right above the irradiated tip; however, substantial silver deposition could be reached on the microstructure tip before the metal deposited on the substrate blocked the laser light. At the same time, it was critical to avoid metal deposition on the spherical parts of microstructures (used for trapping). For this, the structures were equipped with protective shields shown in Figure $2 \mathrm{~d}$ (see also Figure 3). These shields efficiently restricted metal particle access to microstructures. The 
upside-down arrangement avoided uncontrolled settling of large silver particles from the bulk solution to the microstructure surface. The setup allowed precise detection of the transmitted laser light. The power of the laser beam passing through the sample was measured by a power meter (Ophir Optronics Solutions, PD300-3W-V1). Thus, the thickness of the deposited silver layers was controlled by stopping the illumination at transmittance values of $20 \%, 30 \%, 40 \%$, $50 \%, 60 \%, 70 \%$ and $80 \%$. The prepared structures were washed with distilled water, dried and stored in a nitrogen atmosphere.

\section{RESULTS AND DISCUSSION}

\section{Optical trap stability}

The developed SERS probes trapped in dual-beam tweezers were positioned at specific locations inside the sample. When moving the microstructures in the buffer solution, the drag forces exerted by the medium were compensated by optical forces. The friction was minimized when the microstructure traveled along its axis. It was, however, critical to move the structures in the perpendicular direction, where the friction was the highest possible and the arising torque was to be canceled off, too. All this limited the maximal transport velocity at given laser power used for trapping.

The time-sharing dual optical trap was optimized for microstructure transport by setting unequal laser power (duty time) for the two traps. To treat the phenomena theoretically, the microstructures were approximated by a rigid assembly of three identical spheres connected through thin rods of negligible diameter (see Figure 4). The ratio of the distance between the two trapping spheres to the overall length of the structure was assigned by $\beta$. For the sake of 
simplicity, we only considered constant velocity motion in the direction perpendicular to the microstructure axis and in parallel to the microscope slide. In first approximation the optical forces acting in the two traps (see $\boldsymbol{F}_{1}$ and $\boldsymbol{F}_{2}$ in Figure 4) are proportional to the displacements $\left(\boldsymbol{y}_{1}\right.$ and $\left.\boldsymbol{y}_{2}\right)$ of the microstructure's spherical parts from the laser beam centers: $\boldsymbol{F}_{1}=-A_{0} \alpha \boldsymbol{y}_{1}$ and $\boldsymbol{F}_{2}=-A_{0}(1-\alpha) \boldsymbol{y}_{2}$, where $A_{0}$ is the trap stiffness corresponding to the total laser power. $\alpha$ and $(1-\alpha)$ are the percentages of the total laser power belonging to trap 1 and trap 2, respectively. In small tilt angle approximation, the equilibrium equations for zero net force and zero net torque can be solved for $\boldsymbol{y}_{1}$ and $\boldsymbol{y}_{2}$, resulting:

$$
\begin{aligned}
& y_{1}=\frac{F_{0}(2 \beta-1)}{A_{0} \alpha \beta} \\
& y_{2}=\frac{F_{0}(1+\beta)}{A_{0}(1-\alpha) \beta}
\end{aligned}
$$

$F_{0}$ represents the drag force exerted on a single spherical component. Two different situations are depicted in the upper part of Figure 4. A microstructure with $\beta<0.5$ is shown on the left. In this case the torque exerted by the drag forces can only be compensated when the two optical forces act in opposition. This is unfavorable, as $\boldsymbol{F}_{1}$ acts in the direction opposite to the motion. Moreover, the microstructure has to be tilted (compared to the stationary state) during the transfer. The situation changes, when $\beta>0.5$ and both $\boldsymbol{F}_{1}$ and $\boldsymbol{F}_{2}$ point in the direction of motion. In this case the trap can be optimized for zero tilt angle by setting the right laser power ratio in the two traps, i.e. the value of $\alpha$ fulfilling condition: $y_{1}=y_{2}$. 

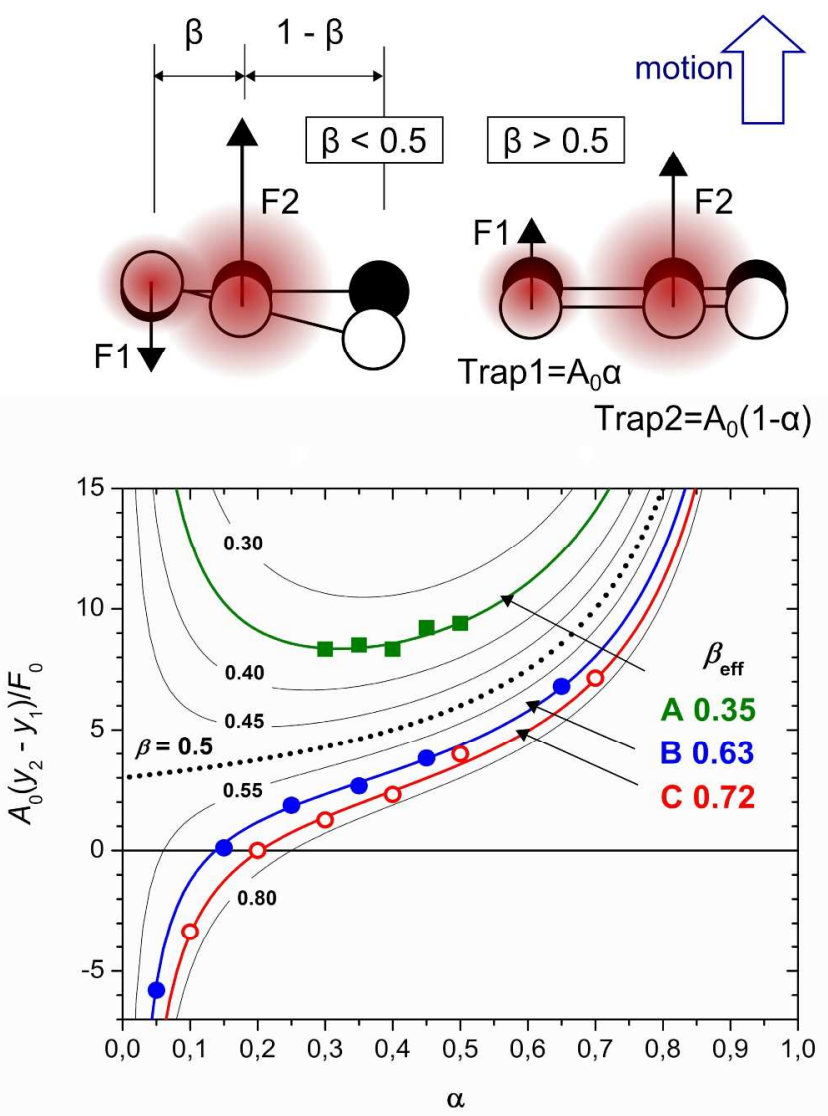

Figure 4. Upper part: Optical forces $\left(\boldsymbol{F}_{1}\right.$ and $\left.\boldsymbol{F}_{2}\right)$ acting in a dual beam optical trap on microstructures (composed of three connected spheres) during transfer in the direction perpendicular to the microstructure axis. The two laser beams are indicated in red (top view). The solid and open assemblies represent microstructures at rest and during motion, respectively. Lower part: Results of theoretical calculations for the microstructure tilt (given by $y_{2}-y_{1}$, see the text) shown together with the experimental data obtained for Type-A (solid squares), Type-B (solid circles) and Type-C (open circles) microstructures.

The obtained theoretical results were validated experimentally by quantifying the tilt of different microstructures during the transfer. Constant velocity motion was induced by moving the piezo-stage. The equilibrium microstructure position (tilt in the trap) was monitored by video 
tracking, and the difference values of $y_{2}-y_{1}$ were determined for various $\alpha$ settings. The obtained data were compared with the theoretical $\alpha$-dependence of $y_{2}-y_{1}$ as calculated from (1) and (2). The corresponding curves are plotted against $\alpha$ (for selected $\beta$ values) in Figure 4. To account for the non-ideal shapes of the used microstructures (other than the theoretical assumption) and the unknown ratio of $F_{0} / A_{0}$ (see (1) and (2)), the measured data were multiplied with a constant so that the obtained points fitted one of the theoretical curves the best. This way an effective $\beta$ was determined for each microstructure type studied.

It can be seen from Figure 4 that Type-B microstructures can be transferred without tilt (i.e. $y_{2}-$ $y_{1}=0$ ) when using $\alpha$ of 0.15 . The corresponding $\beta_{\text {eff }}$ is about 0.63 . By contrast, Type-A microstructures can be characterized by $\beta_{\text {eff }}=0.35$. In this case the tilt is unavoidable during the transfer. Finally, it is shown that an effective $\beta$ (of 0.72 ) can be assigned also to Type-C microstructures even if these are not composed of three spherical parts.

As the larger aspect ratio $\beta$ (i.e. larger distance between the trapping spheres) gives higher stability in the trap the dual beam optical tweezers was set to work with type-B and type-D microstructures in the remaining part of the paper.

\section{SERS measurements}

The quality of silver layers deposited to the microstructures was tested by measuring SERS spectra of emodin $\left(1.10^{-6} \mathrm{M}\right)$ added to the buffer solution in the optical trap experiment. Emodin is an anti-cancer drug that was used previously by the Petrov group for testing optically trappable SERS probes. ${ }^{7,19}$ The excitation laser power $(488 \mathrm{~nm})$ was set to $0.02 \mathrm{~mW}$. Emodin SERS spectra measured with spherical-tip (structure B) and pointed-tip (structure D) structures are shown in Figure 5a together with background spectra detected in the bulk emodin solution (curve 1) and with uncoated microstructures (curve 2). It can be seen, that the Raman signal was only 
detectable in the presence of silver nano-particles, showing the importance of plasmonic effects for molecules adsorbed to the silver surface. There was no evidence of emodin photo-bleaching throughout the experiments. By contrast, the SERS intensity tended to increase gradually when the excitation laser irradiated the deposited silver layer (as it is discussed in more details in the next section).

In general, the spatial resolution of SERS measurements can be maximized when working with pointed tips. On the other hand, the available active SERS area is larger on the spherical tips which can be advantageous when the spatial resolution is of no concern. Indeed, the signal on the spherical-tip (type-B) microstructure was about two times stronger than the one measured with the pointed-tip (type-D) SERS probe - see Figure 5a. The observed difference can be attributed to a geometrical factor. The spot size of the used excitation beam exceeded the diameter of the pointed tip, thus the illuminated active SERS area was smaller here resulting in a weaker signal.

To estimate the sensitivity of the photo-reduced silver layers for SERS detection the observed Raman signal was compared with SERS spectra measured on conventional Ag-citrate colloid particles prepared by the Lee \& Meisel method ${ }^{33}$ (aggregated by $\mathrm{KNO}_{3}$ at final concentration of $\left.2.10^{-2} \mathrm{M}\right)$. The analytical enhancement factor of these colloids is usually in the range of $1.10^{5}$ $1.10^{6}$ as reported for different molecular dyes ${ }^{14}$ and these colloids were used previously for emodin SERS measurements. ${ }^{34,35}$ The SERS signal of emodin $\left(1.10^{-6} \mathrm{M}\right.$, at $\left.1667 \mathrm{~cm}^{-1}\right)$ detected on micron-sized individual colloid aggregates ${ }^{36}$ settled to the bottom glass (not shown) was found to be 5 times larger (at average) than the one measured on spherical-tip micro-structures at same conditions. It is concluded that in case of emodin the SERS enhancement on the microstructure surface is only slightly lower than the enhancement reached on well-established colloid systems. 


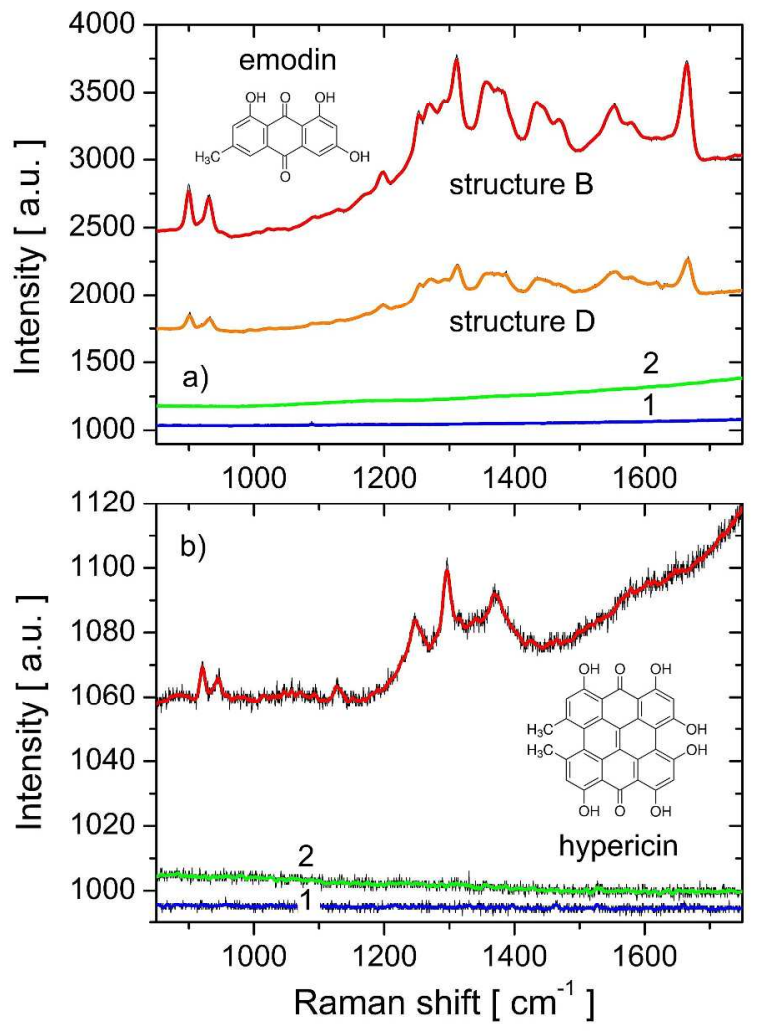

Figure 5. a) SERS spectra of emodin $\left(1.10^{-6} \mathrm{M}\right)$ dissolved in water (containing $1 \% \mathrm{v} / \mathrm{v}$ DMSO and $0.01 \% \mathrm{v} / \mathrm{v}$ Triton-X100) as measured by spherical-tip (type-B) and pointed-tip (type-D) microstructures having silver coatings of $40 \%$ transmittance. Excitation: $0.02 \mathrm{~mW}, 488 \mathrm{~nm}$. Accumulation time: 60 s. b) SERS spectrum of hypericin $\left(1.10^{-5} \mathrm{M}\right)$ detected with type-B microstructure of $60 \%$ layer transmittance. Excitation: $0.006 \mathrm{~mW}, 532 \mathrm{~nm}, 60 \mathrm{~s}$ accumulation. The figure insets show the chemical structure of emodin and hypericin. The curves indicated by 1 and 2 represent background spectra detected in the bulk solution and with uncoated microstructures, respectively.

The new structures were also tested for detection of the naturally occurring photosensitizer hypericin, which is studied extensively for its possible application in photodynamic therapy. ${ }^{37-39}$ 
A SERS spectrum of hypericin excited with the $532 \mathrm{~nm}(0.006 \mathrm{~mW})$ laser is shown in Figure $5 \mathrm{~b}$. The observed detection sensitivity is lower here (as compared to emodin) which can be partially due to reduced adsorption affinity of hypericin to the used silver surfaces.
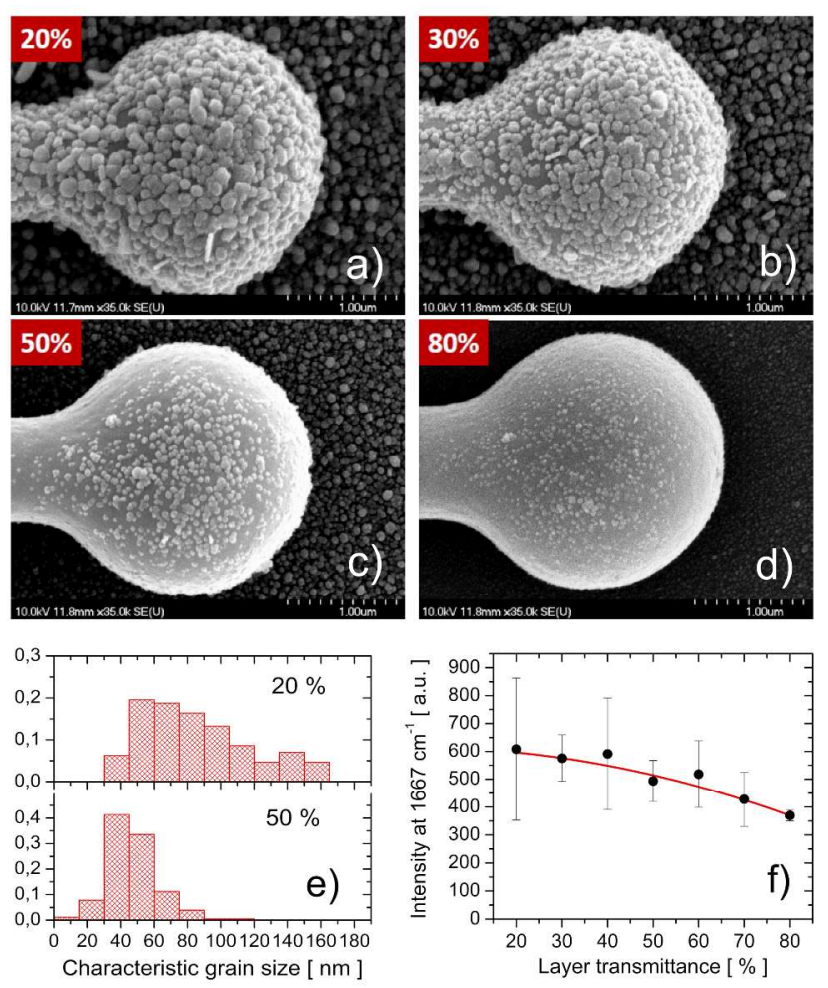

Figure 6. a)-d) SEM pictures of type-B microstructure tips after photo-reduction with final layer transmission of $20,30,50$ and $80 \%$. e) Characteristic diameter distribution of silver nanoparticles on microstructure surfaces evaluated for layer transmissions of 20 and $50 \%$. f) Intensity of the emodin $\left(1.10^{-6} \mathrm{M}\right)$ SERS signal at $1667 \mathrm{~cm}^{-1}$ as a function of silver layer transmission. Each point represents an average of seven consecutive measurements on single microstructures (excitation: $0.02 \mathrm{~mW}, 488 \mathrm{~nm}$; accumulation time: $60 \mathrm{~s}$ ); the error bars indicate the scattering (standard deviation) of the measured intensities.

The photo-reduction process was tested in detail in a series of experiments controlling the transmittance of the photo-reduced silver layer. SEM pictures of type-B microstructures with 
silver layers of different transmittance are shown in Figures 6 a-d. The photo-reduction process produced silver layers of grainy structure both at the glass and the microstructure surface. The characteristic size of silver nano-particles and the surface coverage of microstructures increased towards the lower layer transmission. Size distribution data of silver grains on the polymer surface, as evaluated from the SEM pictures, are shown in Figure 6e. The average grain diameter was found to be about $47 \mathrm{~nm}$ and $82 \mathrm{~nm}$ for the layers with $50 \%$ and $20 \%$ transmissions, respectively.

Figure 6f shows the intensity of the emodin SERS signal at $1667 \mathrm{~cm}^{-1}$ as measured on structures with silver layer transmission in the range of $20-80 \%$. In spite of significant differences in layer characteristics (grain diameter and surface coverage), only a weak signal increase was observed towards lower silver layer transmission values. This behavior was possibly due to exceeding the optimal nano-particle diameter for maximal SERS enhancement at low-transmission layers (see e.g. Ref. 40) the effect of which was compensated by the increased surface coverage. More detailed analysis of all the parameters affecting the SERS intensity would be needed for satisfactory explanation of the observed dependence, which is beyond the scope of the present work. Finally, it is noted that in spite of working with protective shields (Figure 2d), unwanted silver deposition was observed on the microstructure trapping spheres below 30\% transmission of the deposited silver layer.

\section{The effect of illumination}

As mentioned in the previous section, the intensity of the emodin SERS signal tended to increase gradually between consecutive measurements when the excitation laser (488 $\mathrm{nm}$, $0.02 \mathrm{~mW}$ ) was continuously shining on the surface. More detailed analysis of the measured spectra revealed that the character of the measured SERS spectra was also changed. In Figure 7a 
the very first and the seventh emodin SERS spectrum (60 sec accumulation each) is shown as detected on a type-B microstructure with a $60 \%$ transmission silver coating. It can be seen that the extent of the intensity increase is not equal for all the Raman peaks. Figure $7 \mathrm{~b}$ shows the increase of the SERS signal at $1667 \mathrm{~cm}^{-1}$ and the intensity ratio changes of the 931 and $900 \mathrm{~cm}^{-1}$ Raman bands during the measurements.

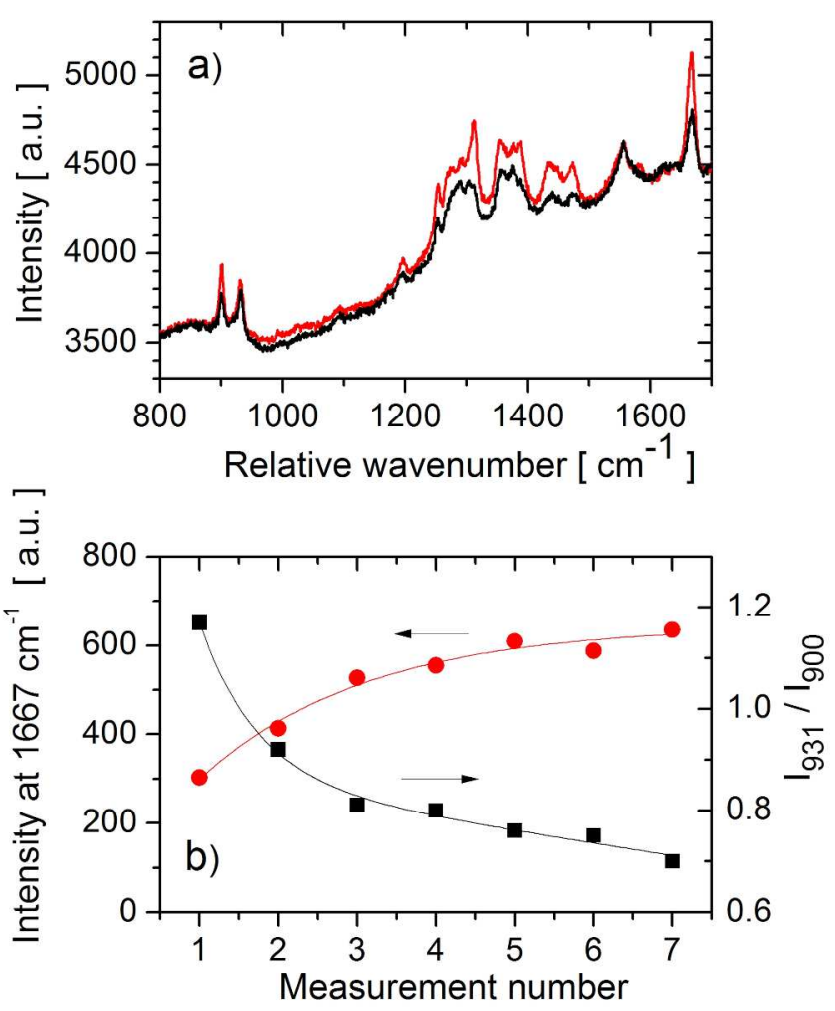

Figure 7. a) SERS spectra of emodin $\left(1.10^{-6} \mathrm{M}\right)$ dissolved in water (containing $1 \% \mathrm{v} / \mathrm{v}$ DMSO and $0.01 \% \mathrm{v} / \mathrm{v}$ Triton-X100) measured on a type-B microstructure with a $60 \%$ transmission silver coating. The black and red spectra belong to the first and seventh acquisition (60 sec accumulation time each) detected after turning the $488 \mathrm{~nm}$ excitation beam on. b) The intensity of the $1667 \mathrm{~cm}^{-1}$ Raman band of emodin and the intensity ratio of the 931 and $900 \mathrm{~cm}^{-1}$ bands as evaluated from consecutive $(60 \mathrm{sec})$ measurements. 
The $\mathrm{pH}$ of the aqueous solution used in our experiments was measured to be 7.6. This value was close to the $\mathrm{p} K_{\mathrm{a}}$ constant for emodin deprotonation, reported to be $7.5,{ }^{35}$ or $8.0 .^{41}$ It follows that at our conditions, emodin molecules were present in the bulk solution both in neutral and anionic states. The spectra of Figure 7a were compared with those reported in Ref. 35 for emodin in Ag-citrate colloids at pH 6 and pH 10 and also in Ref. 34 for $\mathrm{pH}$ values of 4, 7 and 12. Based on this comparison it was concluded that the first (black) spectrum of Figure 7a corresponds to emodin molecules that are predominantly in neutral form, while the other (red) spectrum, measured as the seventh one after turning the excitation beam on, belongs to emodin molecules that are predominantly deprotonated. The intensity ratio changes depicted in Figure $7 \mathrm{~b}$ correlate well with these assumptions. It follows that the $488 \mathrm{~nm}$ laser irradiation changed the local conditions at the silver surface in a way that favored the adsorption of anionic emodin molecules. Silver nano-particles prepared in the presence of citrate usually carry a negative surface charge ${ }^{36}$ which may hinder adsorption of emodin anions by electrostatic repulsion. Light-stimulated reduction of silver nano-particle negative surface charge (caused by photo-effect) was reported. ${ }^{42}$ It is speculated that the same effect was responsible for the changes in surface charge and, in our case, the anionic emodin adsorption onto the microstructures. This was reflected in modified SERS spectra upon laser irradiation.

\section{CONCLUSIONS}

The present paper is a proof-of-concept for fabrication of optically trappable SERS probes where the SERS detection is spatially separated from the trapping positions. The major advantage of this concept is that the SERS-active metal layer and the Raman excitation beam can be optimized independently of the trapping laser parameters. Optically trappable microstructures were prepared by laser-assisted two-photon polymerization of SU-8 photoresist. Photo-reduction 
of silver from silver nitrate solutions was successfully tested for localized deposition of SERSactive silver layers onto the microstructure surface. It was shown that silver nano-particles of different sizes can be prepared on the polymer surface by changing the parameters of the photoreduction process. The new probes were tested by measuring SERS spectra of emodin. Detailed analysis of the obtained spectral characteristics revealed that illumination of the silver nanoparticles by the $488 \mathrm{~nm}(0.02 \mathrm{~mW}$ continuous power) excitation beam changes the local surface conditions in a way that favors the adsorption of anionic emodin molecules.

The SERS micro-probes presented in this work can be applied in different microfluidic devices for targeted SERS measurements. Most of the previous works combining the SERS method with microfluidics focuses on detection of low concentration analytes in solutions. ${ }^{43,44}$ By contrast, the present probes give one an opportunity to move the SERS sensor to specific location inside of the sample. Moreover, the new SERS probes can be applied as disposables that are used for repeated measurements inside of the same microfluidic experiment. In this case the probes can be consecutively injected and transported to the area of interest.

Mobile SERS probes with pointed and spherical tips were studied in this work; however, tips of arbitrary shape can be prepared by the two-photon polymerization process. This way the SERS probes can be fitted to specific applications.

\section{AUTHOR INFORMATION}

\section{Corresponding Author}

* gregor.bano@upjs.sk

ACKNOWLEDGMENTS 
Authors wish to thank Sergei Kruglik for his help with Raman measurements. This work was supported by the APVV-0242-11 grant of the Slovak Ministry of Education and the FP7 EU CELIM 316310 project. This work was also supported by the projects SEPO-II (26220120039) and NanoBioSens $(26220220107,50 \%)$ of the Operation Programme Research and Development funded by the European Regional Development Fund. The work was also supported by the Hungarian Science Research Fund (OTKA grant nos. NN 102624 and NN 114692). L.K. was supported by the Bolyai János Research Scholarship of the Hungarian Academy of Sciences.

\section{REFERENCES}

(1) Svedberg, F.; Li, Z.; Xu, H.; Käll, M. Creating hot nanoparticle pairs for surfaceenhanced Raman spectroscopy through optical manipulation. Nano Lett. 2006, 6, 26392641.

(2) Messina, E.; Cavallaro, E.; Cacciola, A.; Saija, R.; Borghese, F.; Denti, P.; Fazio, B.; D'Andrea, C.; Gucciardi, P. G.; Iati, M. A.; Meneghetti, M.; Compagnini, G.; Amendola, V.; Marago, O. M. Manipulation and Raman Spectroscopy with Optically Trapped Metal Nanoparticles Obtained by Pulsed Laser Ablation in Liquids. J. Phys. Chem. C 2011, 115, 5115-5122.

(3) Tong, L.; Righini, M.; Ujue Gonzalez, M.; Quidant, R.; Käll, M. Optical aggregation of metal nanoparticles in a microfluidic channel for surface-enhanced Raman scattering analysis. Lab Chip 2009, 9, 193-195. 
(4) Jordan, P.; Cooper, J.; McNay, G.; Docherty, F. T.; Smith, W. E.; Sinclair, G.; Padgett, M. J. Three-dimensional optical trapping of partially silvered silica microparticles. Opt. Lett. 2004, 29, 2488-2490.

(5) McNay, G.; Docherty, F. T.; Graham, D.; Smith, W. E.; Jordan, P.; Padgett, M.; Leach, J.; Sinclair, G.; Monaghan, P. B.; Cooper, J. M. Visual observations of SERRS from single silver-coated silica microparticles within optical tweezers. Angew. Chem. Int. Edit. 2004, 43, 2512-2514.

(6) Gessner, R.; Winter, C.; Rosch, P.; Schmitt, M.; Petry, R.; Kiefer, W.; Lankers, M.; Popp, J. Identification of biotic and abiotic particles by using a combination of optical tweezers and in situ Raman spectroscopy. Chemphyschem 2004, 5, 1159-1170.

(7) Balint, S.; Kreuzer, M. P.; Rao, S.; Badenes, G.; Miskovsky, P.; Petrov, D. Simple Route for Preparing Optically Trappable Probes for Surface-Enhanced Raman Scattering. J. Phys. Chem. C 2009, 113, 17724-17729.

(8) Rao, S.; Balint, S.; Lovhaugen, P.; Kreuzer, M.; Petrov, D. Measurement of Mechanical Forces Acting on Optically Trapped Dielectric Spheres Induced by Surface-Enhanced Raman Scattering. Phys. Rev. Lett. 2009, 102, 087401.

(9) Meng, W.; Hu, F.; Jiang, X.; Lu, L. Preparation of Silver Colloids with Improved Uniformity and Stable Surface-Enhanced Raman Scattering. Nanoscale Res. Lett. 2015, 10,34 . 
(10) Giorgetti, E.; Marsili, P.; Giammanco, F.; Trigari, S.; Gellini, C.; Muniz-Miranda, M. Ag nanoparticles obtained by pulsed laser ablation in water: surface properties and SERS activity. J. Raman Spectrosc. 2015, 46, 462-469.

(11) Luo, S.; Sivashanmugan, K.; Liao, J.; Yao, C.; Peng, H. Nanofabricated SERS-active Substrates for Single-Molecule to Virus Detection in-vitro: A Review. Biosens. Bioelectron. 2014, 61, 232-240.

(12) Sharma, B.; Cardinal, M.F.; Kleinman, S. L.; Greeneltch, N. G.; Frontiera, R. R.; Blaber, M. G.; Schatz, G. C.; Van Duyne, R. High-Performance SERS Substrates: Advances and Challenges. MRS Bull. 2013, 38, 615-624

(13) Lee, J.; Seo, J.; Kim, D.; Shin, S.; Lee, S.; Mahata, C; Lee, H.; Min, B.; Lee, T. Capillary Force-Induced Glue-Free Printing of Ag Nanoparticle Arrays for Highly Sensitive SERS Substrates, ACS Appl. Mater. Interfaces, 2014, 6, 9053-9060.

(14) Le Ru, E. C.; Etchegoin, P. G. Principles of Surface-Enhanced Raman Spectroscopy: And Related Plasmonic Effects. Elsevier 2009

(15) Cialla, D.; März, A.; Bohme, R.; Theil, F.; Weber, K.; Schmitt, M.; Popp, J. SurfaceEnhanced Raman Spectroscopy (SERS): progress and trends. Anal. Bioanal. Chem. 2012, 403, 27-54.

(16) Yamamoto, Y. S.; Ozaki, Y.; Itoh, T. Recent Progress in the Electromagnetic Mechanism of Surface-Enhanced Raman Scattering. J. Photoch. Photobio. C. 2014, $21,81-104$. 
(17) Schlucker, S. Surface-Enhanced Raman Spectroscopy: Concepts and Chemical Applications. Angew. Chem,. Int. Edit. 2014, 53, 4756-4795.

(18) Rao, S.; Raj, S.; Cossins, B.; Marro, M.; Guallar, V.; Petrov, D. Direct Observation of Single DNA Structural Alterations at Low Forces with Surface-Enhanced Raman Scattering. Biophys. J. 2013, 104, 156-162.

(19) Balint, S.; Rao, S.; Marro Sanchez, M.; Huntosova, V.; Miskovsky, P.; Petrov, D. Diffusion and cellular uptake of drugs in live cells studied with surface-enhanced Raman scattering probes. J. Biomed. Opt. 2010, 15, 027005.

(20) Balint, S.; Rao, S.; Marro, M.; Miskovsky, P.; Petrov, D. Monitoring of local pH in photodynamic therapy-treated live cancer cells using surface-enhanced Raman scattering probes. J. Raman Spectrosc. 2011, 42, 1215-1221.

(21) Seol, Y.; Carpenter, A. E.; Perkins, T. T. Gold nanoparticles: enhanced optical trapping and sensitivity coupled with significant heating. Opt. Lett. 2006, 31, 24292431.

(22) Palima, D.; Banas, A. R.; Vizsnyiczai, G.; Kelemen, L.; Ormos, P.; Gluckstad, J. Wave-guided optical waveguides. Opt. Express 2012, 20, 2004-2014.

(23) Phillips, D. B.; Simpson, S. H.; Grieve, J. A.; Bowman, R.; Gibson, G. M.; Padgett, M. J.; Rarity, J. G.; Hanna, S.; Miles, M. J.; Carberry, D. M. Force sensing with a shaped dielectric micro-tool. Epl-Europhys. Lett. 2012, 99, 58004. 
(24) Aekbote, B. L.; Schubert, F.; Ormos, P.; Kelemen, L. Gold nanoparticle-mediated fluorescence enhancement by two-photon polymerized 3D microstructures. Opt. Mater. 2014, 38, 301-309.

(25) Canamares, M. V.; Garcia-Ramos, J. V.; Gomez-Varga, J. D.; Domingo, C.; SanchezCortes, S. Ag nanoparticles prepared by laser photoreduction as substrates for in situ surface-enhanced raman scattering analysis of dyes. Langmuir 2007, 23, 5210-5215.

(26) Canamares, M. V.; Garcia-Ramos, J. V.; Sanchez-Cortes, S.; Castillejo, M.; Oujja, M. Comparative SERS effectiveness of silver nanoparticles prepared by different methods: A study of the enhancement factor and the interfacial properties. J. Colloid Interf. Sci. 2008, 326, 103-109.

(27) Bjerneld, E. J.; Murty, K.; Prikulis, J.; Kall, M. Laser-induced growth of Ag nanoparticles from aqueous solutions. Chemphyschem 2002, 3, 116-119.

(28) Muniz-Miranda, M. SERS-active Ag/SiO2 colloids: photoreduction mechanism of the silver ions and catalytic activity of the colloidal nanoparticles. J. Raman Spectrosc. 2004, $35,839-842$.

(29) Jurasekova, Z.; del Puerto, E.; Bruno, G.; Garcia-Ramos, J. V.; Sanchez-Cortes, S.; Domingo, C. Extractionless non-hydrolysis surface-enhanced Raman spectroscopic detection of historical mordant dyes on textile fibers. J. Raman Spectrosc. 2010, 41, 1455-1461. 
(30) Jurasekova, Z.; Domingo, C.; Garcia-Ramos, J. V.; Sanchez-Cortes, S. In situ detection of flavonoids in weld-dyed wool and silk textiles by surface-enhanced Raman scattering. J. Raman Spectrosc. 2008, 39, 1309-1312.

(31) Tan, S.; Erol, M.; Attygalle, A.; Du, H.; Sukhishvili, S. Synthesis of positively charged silver nanoparticles via photoreduction of AgNO3 in branched polyethyleneimine/HEPES solutions. Langmuir 2007, 23, 9836-9843.

(32) He, L.; Riassetto, D.; Bouvier, P.; Rapenne, L.; Chaix-Pluchery, O.; Stambouli, V.; Langlet, M. Controlled growth of silver nanoparticles through a chemically assisted photocatalytic reduction process for SERS substrate applications. J. Photoch. Photobiol. A 2014, 277, 1-11.

(33) Lee, P. C.; Meisel, D. Adsorption and Surface-Enhanced Raman of Dyes on Silver and Gold Sols. J. Phys. Chem. 1982, 86, 3391-3395.

(34) Fabriciova, G.; Sanchez-Cortes, S.; Garcia-Ramos, J. V.; Miskovsky, P. Surfaceenhanced Raman spectroscopy study of the interaction of the antitumoral drug emodin with human serum albumin. Biopolymers 2004, 74, 125-130.

(35) Sevilla, P.; Garcia-Blanco, F.; Garcia-Ramos, J. V.; Sanchez-Cortes, S. Aggregation of antitumoral drug emodin on Ag nanoparticles: SEF, SERS and fluorescence lifetime experiments. Phys. Chem. Chem. Phys. 2009, 11, 8342-8348.

(36) Canamares, M. V.; Garcia-Ramos, J. V.; Gomez-Varga, J. D.; Domingo, C.; SanchezCortes, S. Comparative study of the morphology, aggregation, adherence to glass, and surface-enhanced Raman scattering activity of silver nanoparticles prepared by 
chemical reduction of $\mathrm{Ag}+$ using citrate and hydroxylamine. Langmuir 2005, 21, 8546-8553.

(37) Agostinis, P.; Vantieghem, A.; Merlevede, W.; De Witte, P. A. Hypericin in Cancer Treatment: More Light on the Way. Int. J. Biochem. Cell Biol. 2002, 34, 221-241.

(38) Miskovsky, P. Hypericin - a New Antiviral and Antitumor Photosensitizer: Mechanism of Action and Interaction with Biologycal Macromolecules. Curr. Drug Targets. 2002, 3, 55-84.

(39) Saw, C. L. L.; Olivo, M.; Soo, K. C.; Heng, P. W. S. Delivery of Hypericin for Photodynamic Applications. Cancer Lett. 2006, 241, 23-30.

(40) Stamplecoskie, K. G.; Scaiano, J. C.; Tiwari, V. S.; Anis, H. Optimal Size of Silver Nanoparticles for Surface-Enhanced Raman Spectroscopy. J. Phys. Chem. C 2011, $115,1403-1409$.

(41) da Cunha, A. R.; Duarte, E. L.; Teresa Lamy, M.; Coutinho, K. Protonation/deprotonation process of Emodin in aqueous solution and $\mathrm{pK}(\mathrm{a})$ determination: UV/Visible spectrophotometric titration and quantum/molecular mechanics calculations. Chem. Phys. 2014, 440, 69-79.

(42) Popov, A. K.; Tanke, R. S.; Brummer, J.; Taft, G.; Loth, M.; Langlois, R.; Wruck, A.; Schmitz, R. Laser-stimulated synthesis of large fractal silver nanoaggregates. Nanotechnology 2006, 17, 1901-1905.

(43) Wang, C.; Yu, C. Analytical Characterization Using Surface-Enhanced Raman Scattering (SERS) and Microfluidic Sampling. Nanotechnology 2014, 26, 092001. 
(44) White, I. M.; Yazdi, S. H.; Yu, W. W. Optofluidic SERS: Synergizing Photonics and Microfluidics for Chemical and Biologycal Analysis. Microfluid. Nanofluid. 2012, 13, 205-216. 


\section{Page 29 of 37}

Table of Contents Graphic

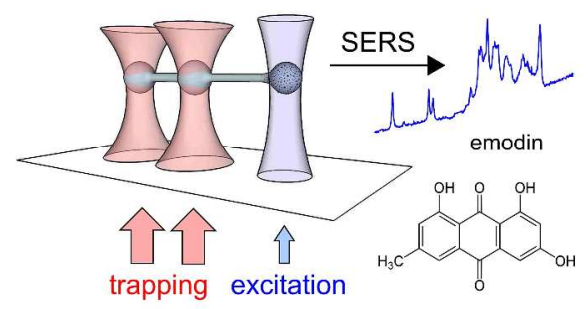

14

15

16

17

18

19

20

21

22

23

24

25

26

27

28

29

30

31

32

33

34

35

36

37

38

39

40

41

42

43

44

45

46

47

48

49

50

51

52

53

54

55

56

57

58

59

60

ACS Paragon Plus Environment 


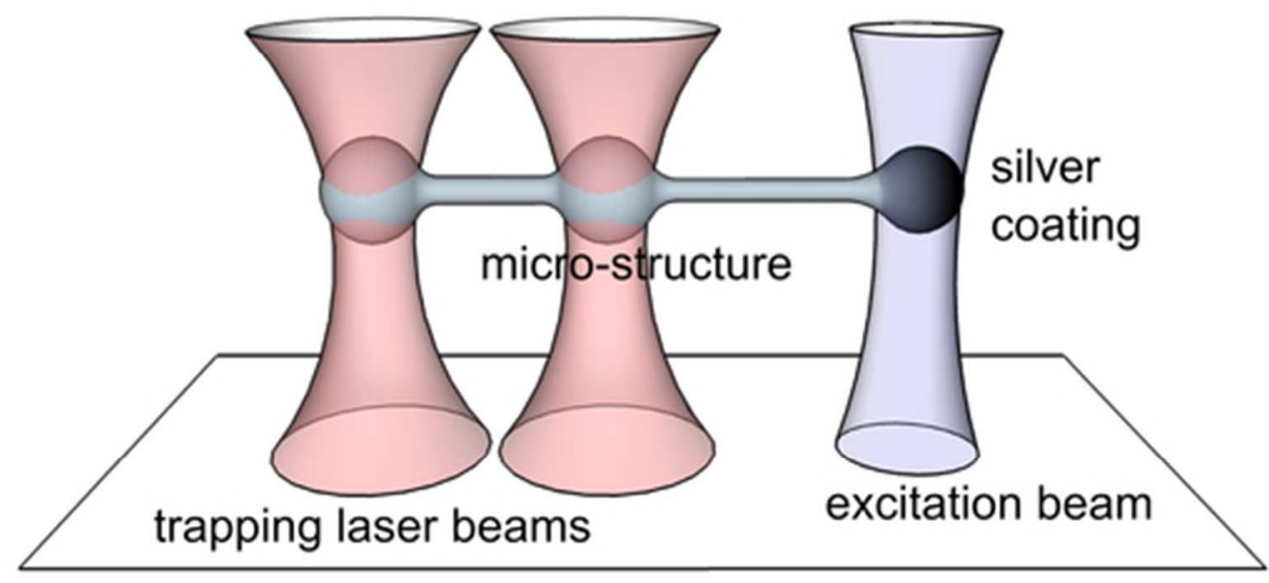

$44 \times 22 \mathrm{~mm}(300 \times 300$ DPI $)$ 

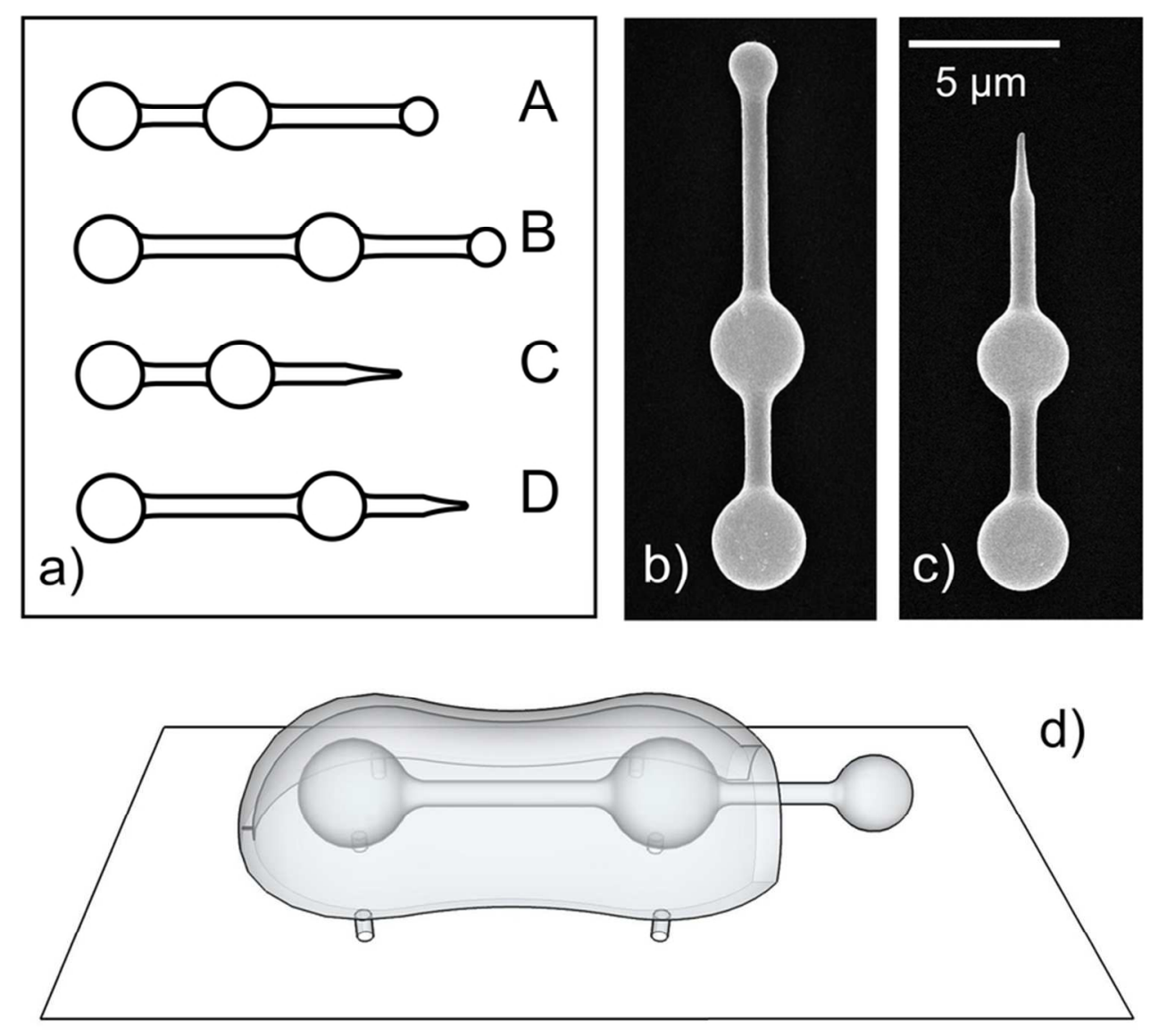

77x70mm (300 x 300 DPI)

39

40

41

42

43

44

45

46

47

48

49

50

51

52

53

54

55

56 

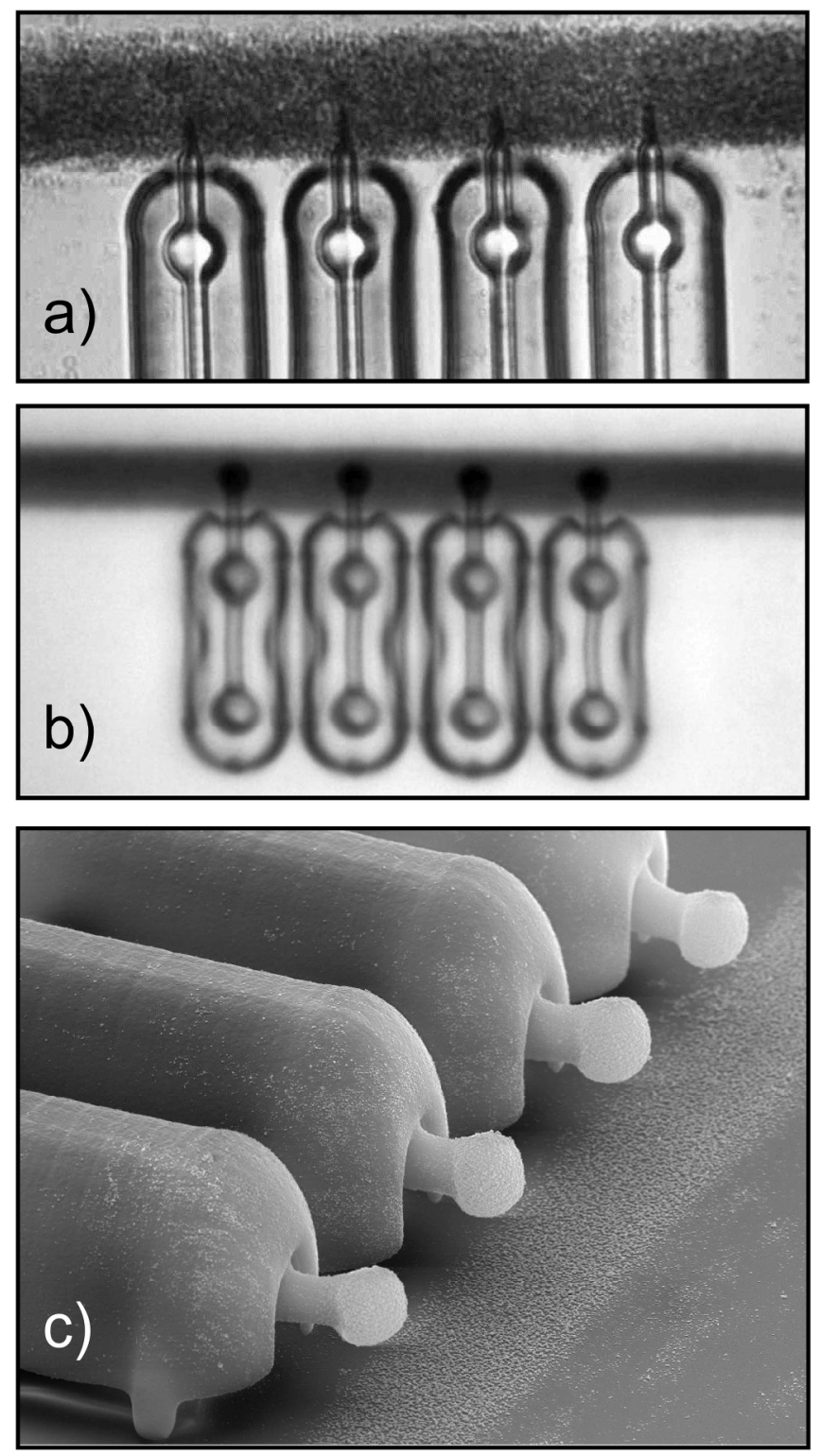

$149 \times 264 \mathrm{~mm}(300 \times 300 \mathrm{DPI})$ 

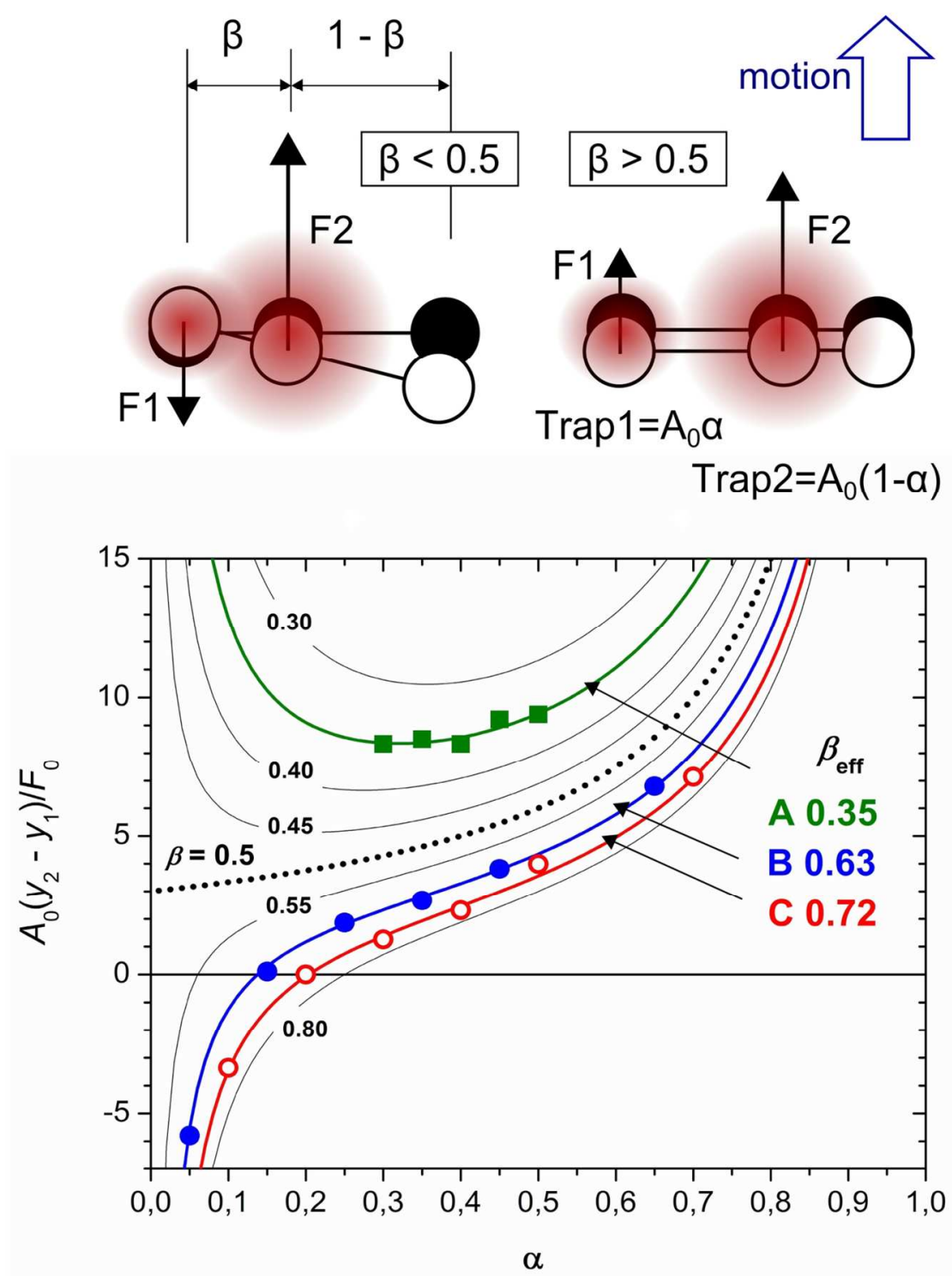

$118 \times 165 \mathrm{~mm}(300 \times 300$ DPI) 


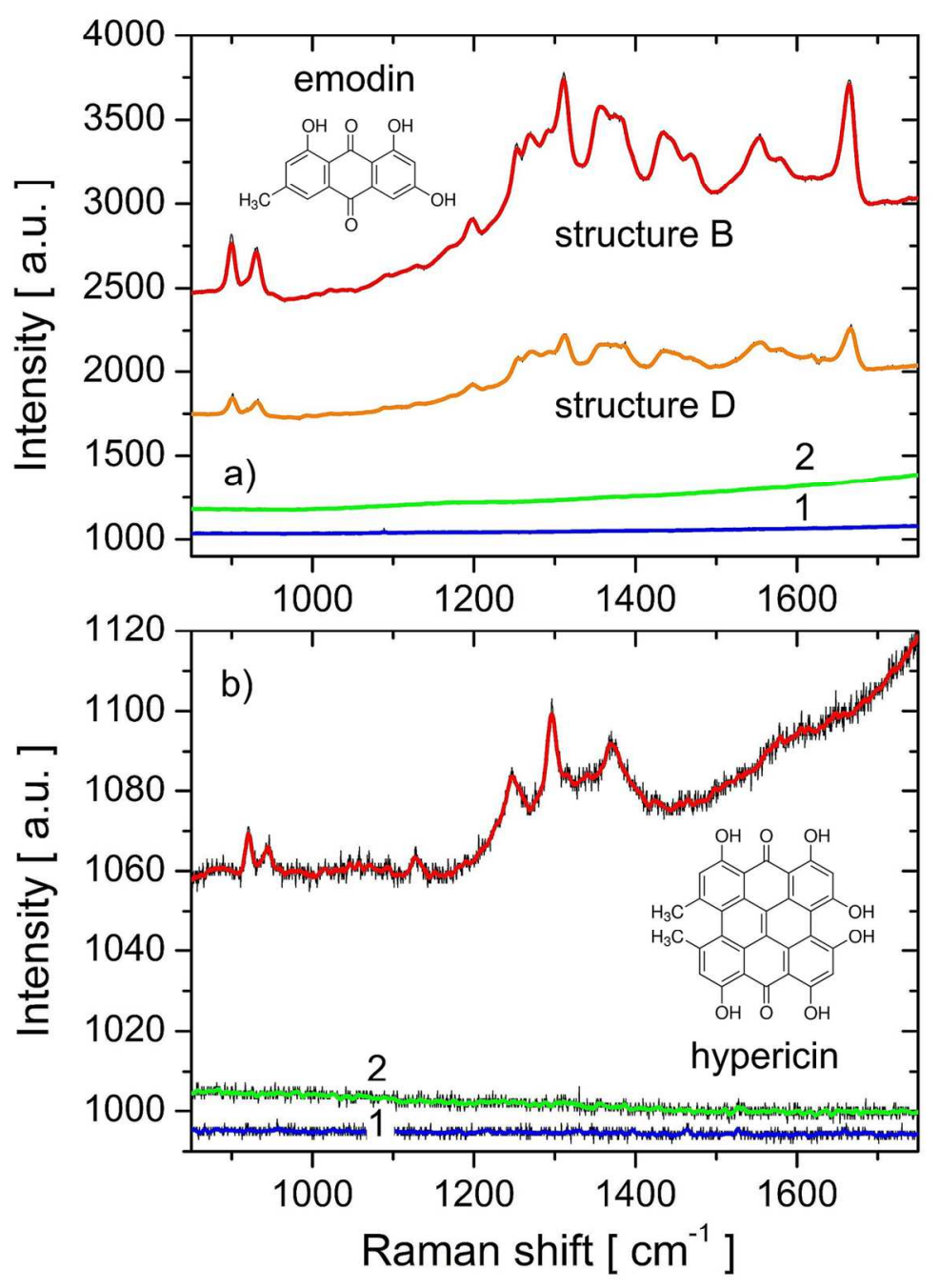

$121 \times 174 \mathrm{~mm}(300 \times 300$ DPI $)$ 

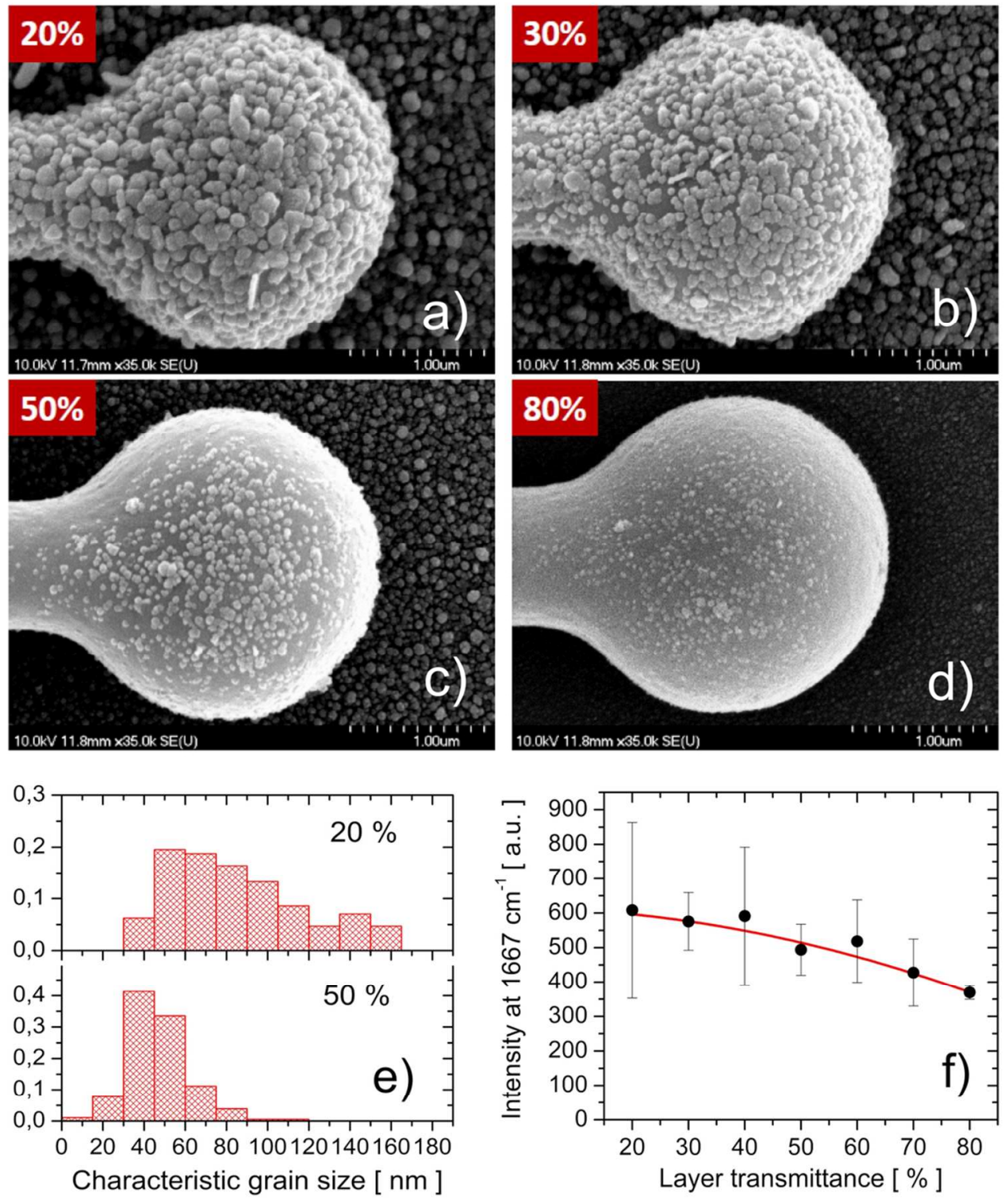

$99 \times 117 \mathrm{~mm}(300 \times 300$ DPI $)$ 

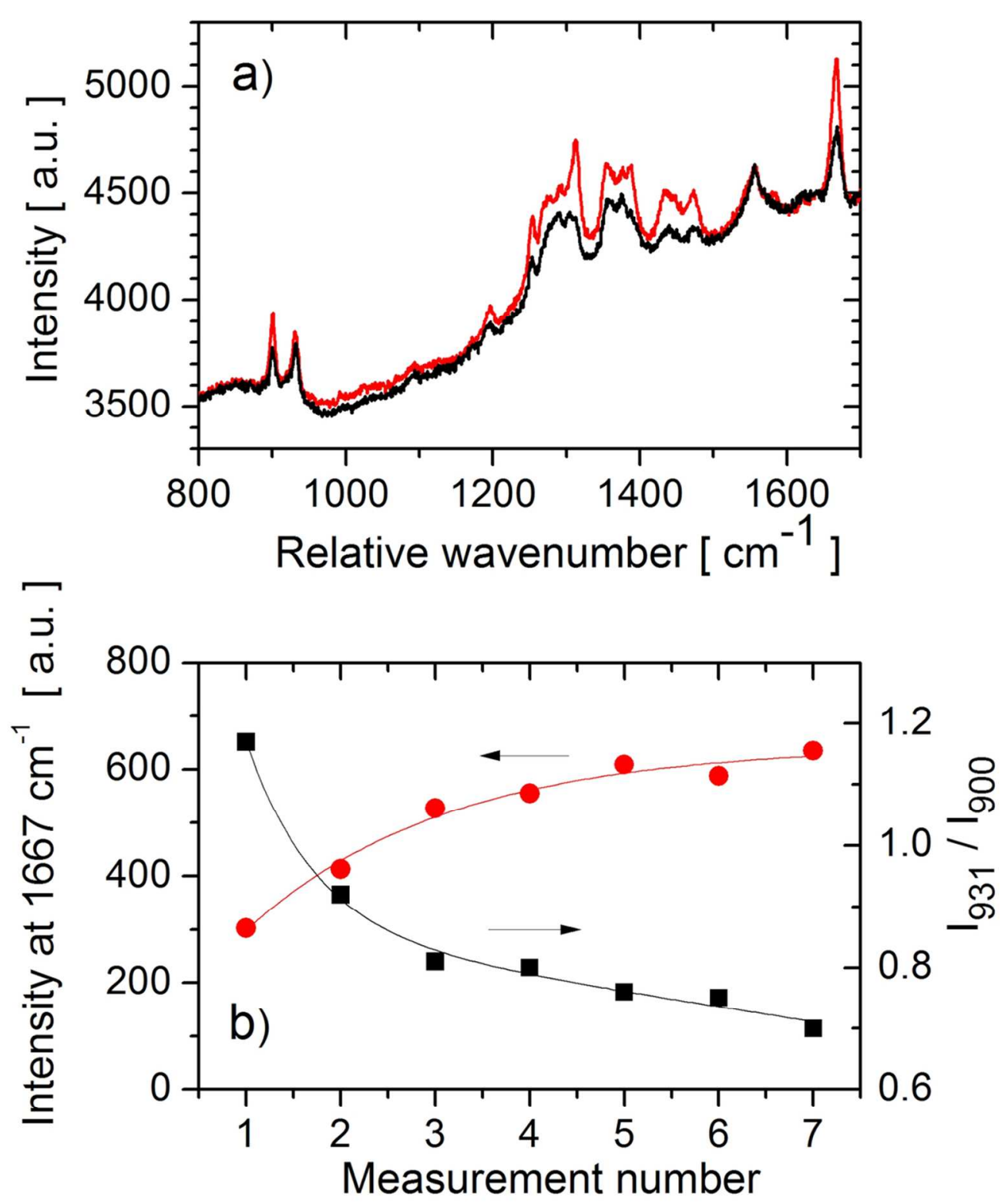

$100 \times 118 \mathrm{~mm}(300 \times 300 \mathrm{DPI})$ 


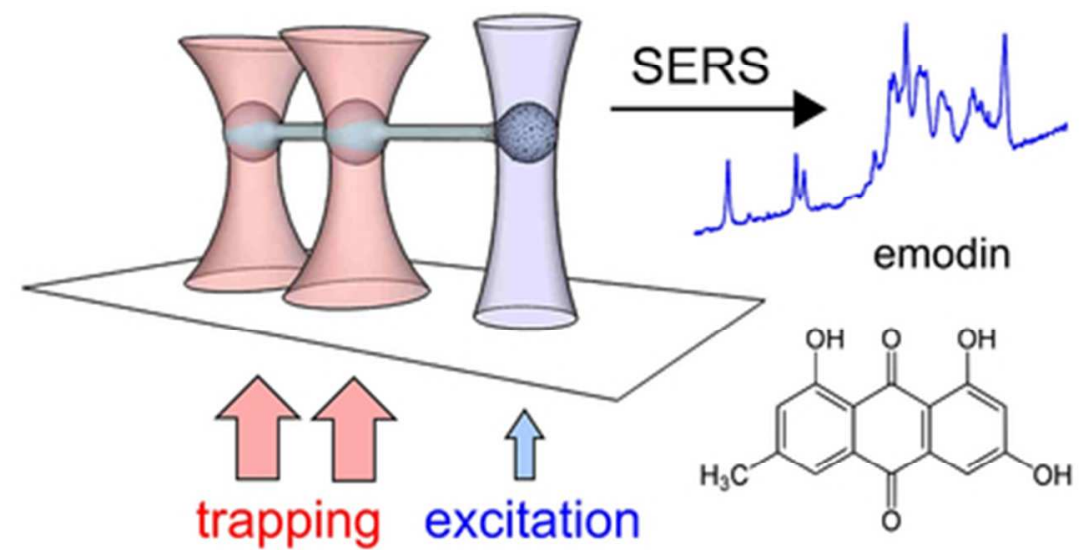

$35 \times 21 \mathrm{~mm}(300 \times 300 \mathrm{DPI})$ 